\title{
Study of the Electrical Resistance of Andisols Subjected to Electro-Remediation Treatment
}

\author{
M.V. Vázquez, ${ }^{\text {a, }}$ F. Hernández-Luis, ${ }^{\text {b }}$ D. Grandoso, ${ }^{\text {b }}$ C.D. Arbelo ${ }^{c}$ \\ ${ }^{a)}$ Instituto de Quimica (Electrochemical Group), Faculty of Exact and Natural Sciences, \\ Universidad de Antioquia, A.A.1226, Medellin, Colombia \\ ${ }^{b)}$ Department of Physical Chemistry, Universidad de la Laguna, Tenerife, España \\ ${ }^{c}$ Department of Soil Science and Geology, Universidad de La Laguna, Tenerife, España
}

Received 1 April 2004; accepted 15 June 2004

\begin{abstract}
Studies of soil resistance suggest that this type of measurement when carried out simultaneously with decontamination treatment, serves as a rapid method for analyzing the behavior of different soils under study, especially when an electric field is not applied. The variation observed in the first step of electro-remediation is related to the ionic content of the segments adjacent to the electrodes. The use of a wash flow facilitates the formation of "ionic batches" near the electrodes. The soil with the higher content of organic material shows lower resistance in the first stage of the treatment. Measuring the electrolysis current without the use of wash flow, together with the other experimental parameters, allows the evaluation of the soil characteristics in experiments of relatively short duration.
\end{abstract}

Keywords: andisols, electrolysis current, electrical resistance, electro-remediation, heavy metals.

\section{Introducción}

La técnica de electro-remediación aplicada a nivel de laboratorio, constituye una herramienta útil para el estudio de los distintos equilibrios que se establecen en el interior de un suelo contaminado [1-8]. Esta utilidad obedece fundamentalmente al hecho de poder recopilar durante el experimento un importante número de parámetros tales como la variación del $\mathrm{pH}$, la conductividad específica, la

\footnotetext{
* Corresponding author: E-mail address: mvazquez@matematicas.udea.edu.co.
} 
concentración de especies contaminantes, la corriente circulante, la resistencia eléctrica del suelo, los potenciales eléctricos generados en distintas zonas del mismo, etc. Estas magnitudes, cuando son analizadas de manera conjunta, permite prever en que magnitud serán movilizados distintos iones contaminantes bajo la acción de un campo eléctrico aplicado directamente en el suelo.

Dentro de los parámetros arriba mencionados, el control de la acidez del suelo resulta especialmente importante en relación con la forma bajo la cual los contaminantes se encontrarán, así como del tipo de interacción que presentarán con las partículas del suelo [5-8].

El grado de acidez, que de forma natural presenta el suelo o el inducido por los mismos contaminantes, puede ser modificado sustancialmente debido al tratamiento de electro-remediación como consecuencia de la producción de $\mathrm{H}_{2}(\mathrm{~g})$ y $\mathrm{OH}^{-}(\mathrm{aq})$ en el cátodo y $\mathrm{O}_{2}(\mathrm{~g})$ y $\mathrm{H}^{+}(\mathrm{aq})$ en el ánodo. Así, las especies cargadas, $\mathrm{H}^{+}$(aq) y $\mathrm{OH}^{-}(\mathrm{aq})$, migrarán creando un frente ácido y uno básico, respectivamente. Además, el movimiento de estos frentes es ayudado por gradientes de concentración que se generan como consecuencia de la difusión [9].

Una de las posibilidades experimentales para reducir la influencia de los frentes ácido y alcalino creados es el empleo de un flujo constante de agua sobre las cámaras de los electrodos. De esta manera es posible remover continuamente los productos de la electrólisis. Por otra parte la eliminación de especies iónicas en las cercanías de los electrodos puede afectar la eficiencia del proceso electroquímico. Una manera de evaluar esto es analizando de que manera se modifica la resistencia eléctrica que ofrece el suelo cuando se le aplica la diferencia de potencial.

En aplicaciones prácticas es importante tener en cuenta los valores de resistividad que presenta un determinado terreno, especialmente desde el punto de vista de su actividad corrosiva de estructuras enterradas. De este modo se realizan estudios integrando las características físicas y químicas de los suelos con los resultados de resistividad para evaluar el comportamiento del material. 
Las medidas de resistividad pueden ser realizadas sobre el terreno para no perturbar el material o en el laboratorio donde se debe controlar los valores de humedad y compactación para tener una muestra representativa de la situación real. En medidas sobre terreno se emplea normalmente el método de Wenner, o de los 4 electrodos. En este procedimiento se emplean 4 barras metálicas separadas una distancia constante y que se hallan situadas sobre una línea recta. Se hace pasar una corriente eléctrica a través de los electrodos exteriores midiendo la diferencia de potencial entre los electrodos interiores [10].

Las medidas de resistencia en suelos resultan de utilidad para realizar estudios para evaluar el grado de contaminación por diversos agentes [11-12]

En el laboratorio puede analizarse la variación de la resistencia de los suelos sometidos a tratamiento electrocinética mediante el monitoreo de la corriente durante el proceso de remediación. En este caso se considera a la porción de suelo bajo estudio como un conductor eléctrico.

En el presente trabajo se presentan los resultados obtenidos al someter a electroremediación a suelos de distintas características, provenientes de la región de Antioquia, Colombia. Los estudios fueron orientados al análisis de la influencia del potencial aplicado, del tipo de suelo y del empleo de soluciones de lavado por las cámaras de los electrodos.

Para comparación se incluyen también variaciones obtenidas en el estudio de suelos de origen volcánico similares, provenientes de la isla de Tenerife, España.

\section{Materiales y métodos}

\section{Suelos estudiados}

Los suelos fueron identificados como Suelo $O$ y Suelo I, respectivamente, y las características principales de los mismos se presentan en la Tabla 1.

Previamente al tratamiento electroquímico, los suelos fueron secados a temperatura ambiente $\left(25^{\circ} \mathrm{C}\right)$ y posteriormente llevados a un tamaño de $20 \mu \mathrm{m}$. 


\section{Test electrocinético}

Para el estudio electrocinético fue empleada una celda como la descrita previamente [5-8]. Esta celda emplea dos electrodos de grafito que actúan como ánodo y cátodo, respectivamente. Para el tratamiento se aplicó un potencial constante entre los electrodos durante 8 horas.

Tabla 1. Características de los suelos estudiados.

\begin{tabular}{lcc}
\hline & Suelo O & Suelo I \\
\hline Arena / \% & 40 & 70 \\
Limo / \% & 40 & 16 \\
Arcilla / \% & 20 & 14 \\
$\mathrm{pH}$ & 5.2 & 5.5 \\
Materia orgánica / \% & 18.1 & 1.0 \\
Capacidad de intercambio efectiva / meq $100 \mathrm{~g}^{-1}$ & 16.3 & 16.4 \\
\hline
\end{tabular}

\section{Test electrocinético}

Para el estudio electrocinético fue empleada una celda como la descrita previamente [5-8]. Esta celda emplea dos electrodos de grafito que actúan como ánodo y cátodo, respectivamente. Para el tratamiento se aplicó un potencial constante entre los electrodos durante 8 horas.

Como se indicó antes, las cámaras de los electrodos poseen sendas entradas y salidas que permiten mantener un flujo continuo de la solución de lavado que en este caso fue agua destilada.

Para contaminar los suelos se empleó una disolución $0.1 \mathrm{M}$ de nitrato de cadmio y nitrato de plomo en agua destilada. Las siguientes relaciones (masa suelo : volumen solución) fueron empleadas: Suelo $O$ 1:0.5 y Suelo I 1:0.3, respectivamente; una vez realizada la mezcla, se dejó en contacto durante las 24 horas previas al experimento electroquímico. Las distintas relaciones empleadas para cada suelo obedecen a la necesidad de preparar mezclas de similar consistencia. En los experimentos con soluciones de lavado se mantuvo un flujo medio de agua destilada de $130 \mathrm{~mL} / \mathrm{h}$, aproximadamente. 


\section{Tratamiento posterior a la electro-remediación}

Una vez finalizado el tratamiento electroquímico, el suelo fue segmentado en cinco fragmentos similares. Cada uno de estos fragmentos fue suspendido en agua destilada en una relación 1:1 (masa fragmento: volumen agua) durante 16 horas. El pH y la conductividad específica de estas suspensiones fueron medidos directamente. Posteriormente, las suspensiones fueron filtradas para determinar el contenido de metal pesado libre.

Análisis similares se realizaron directamente en las soluciones de lavado para los experimentos que emplearon flujo de estas soluciones por las cámaras de los electrodos.

\section{Resultados}

\section{Comportamiento general}

Cuando se analiza la variación de la resistencia en el suelo con el tratamiento electroquímico a tiempos relativamente largos (192 horas), se obtiene un comportamiento como el que se muestra en la Fig. 1.

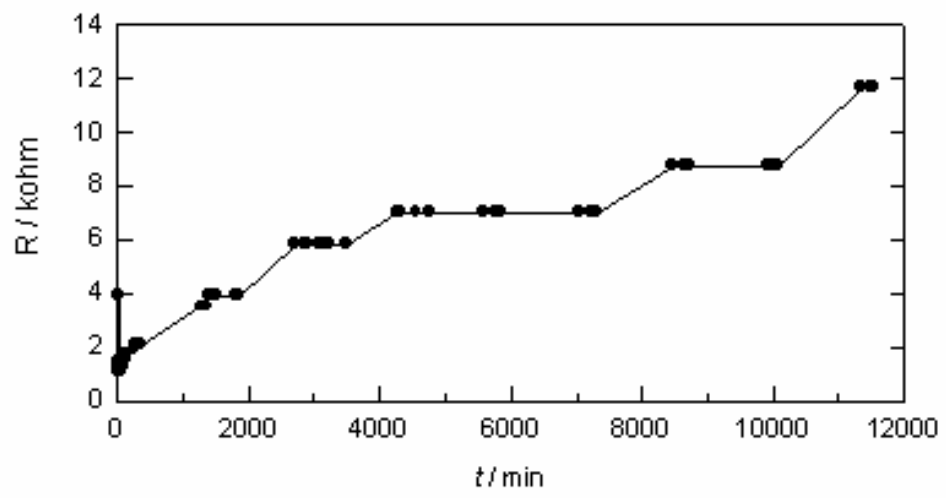

Figura 1. Variación de la resistencia eléctrica con el tiempo. Suelo O, sin lavado en las cámaras.

En este caso se presenta la variación correspondiente al Suelo $O$ en un experimento donde no se aplica el lavado continuo de las cámaras de los electrodos. Después de una caída inicial de la resistencia, se alcanza un valor 
estable a las 66 horas de tratamiento, aproximadamente, para luego aumentar de nuevo sobre las 130 horas. Cada una de estas zonas de resistencia se corresponde con procesos que se establecen dentro del suelo bajo tratamiento.

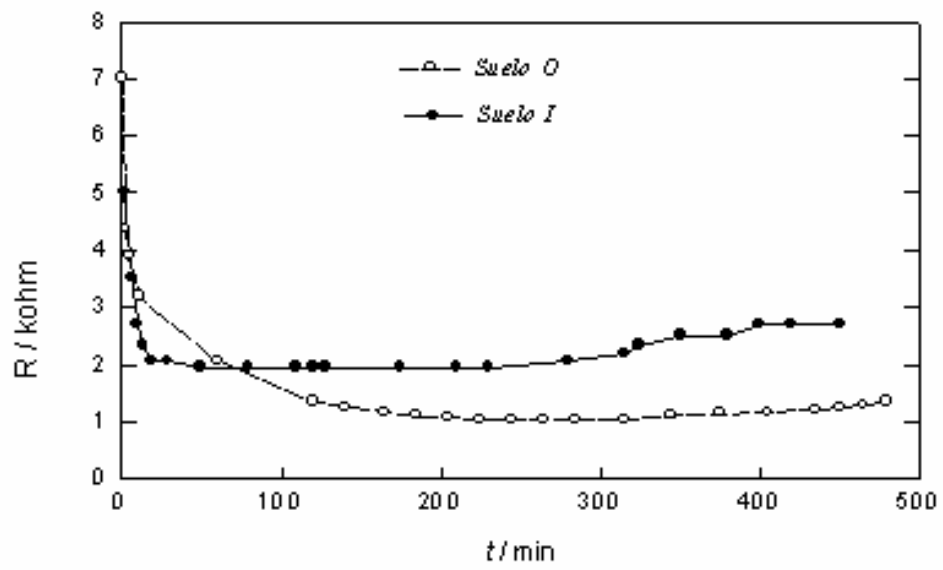

Figura 2. Variación de la resistencia eléctrica de los suelos estudiados en función del tiempo de electrólisis, durante un experimento corto.

Dado que se trata de evaluar parámetros que permitan sacar conclusiones sobre el comportamiento de los suelos, para experimentos de laboratorio de tiempos de electrólisis cortos, se centró el análisis en las primeras 8 horas de tratamiento. En la Fig. 2 se muestran los resultados de la resistencia obtenidos con los dos suelos estudiados. Se observa que inicialmente el Suelo $O$ presenta una mayor resistencia al paso de la corriente, pero esta tendencia se invierte luego de los 100 minutos de electrólisis.

\section{Variación del campo eléctrico aplicado}

En la Fig. 3 se presenta la variación de la resistencia eléctrica para el Suelo I cuando se aplican dos valores diferentes de campo eléctrico. En este caso, la aplicación de un potencial de $35 \mathrm{~V}$ entre los electrodos provoca que inicialmente se tenga un elevado valor de resistencia, que al cabo de unos 100 minutos de tratamiento alcanza un valor más o menos estable hasta el final del experimento. 
Por otra parte, al menor voltaje aplicado se observa una variación menos acentuada de la resistencia durante el tratamiento electroquímico.

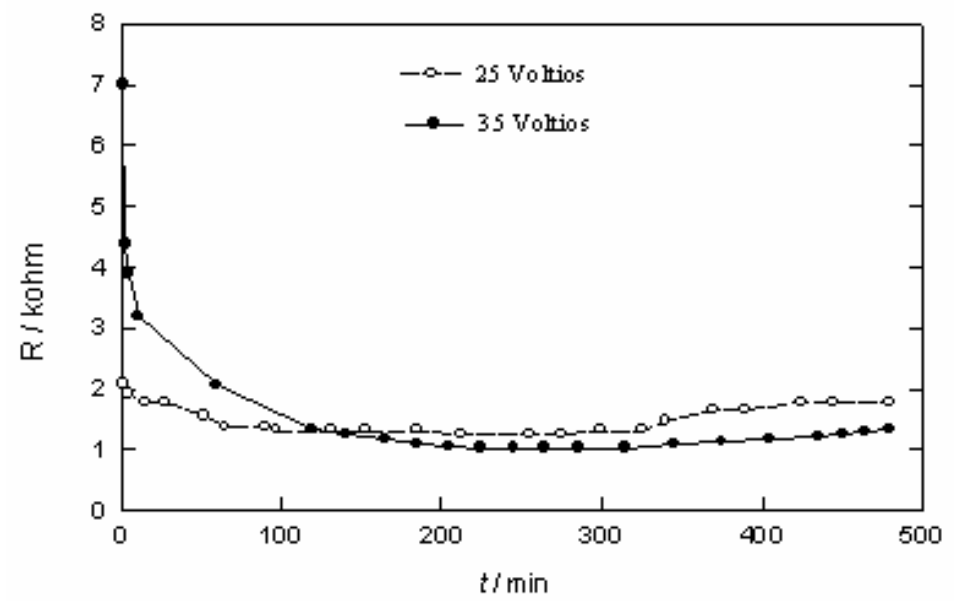

Figura 3. Variación de la resistencia eléctrica del Suelo I en función del tiempo de electrólisis, para dos potenciales aplicados.

\section{Influencia del tipo de suelo bajo estudio}

Cuando se comparan los resultados obtenidos con los dos suelos bajo estudio se obtienen los resultados mostrados en la Fig. 4. Se analiza también el efecto del flujo de las soluciones de lavado en las cámaras de los electrodos. En ambos casos se obtiene una variación más regular cuando no se emplean soluciones de lavado.

\section{Discusión}

Para la correcta interpretación de las variaciones observadas debe ser tenido en cuenta como varían otros parámetros durante el tratamiento de remediación electroquímica. Dentro de estos últimos deben mencionarse la conductividad especifica y el $\mathrm{pH}$ en las distintas regiones del suelo, así como la concentración de iones metálicos contaminantes libres. Teniendo presente los valores de estos parámetros obtenidos para los dos suelos [13], así como las características fisicoquímicas de los mismos, pueden hacerse las siguientes consideraciones:

En los primeros 30 minutos de tratamiento, los distintos valores de resistencia obtenidos podrían ser resultado de la diferente capacidad reguladora del $\mathrm{pH}$ que muestran estos suelos. De este modo, en el Suelo I, que presenta la menor 
capacidad reguladora (reflejado en los altos valores de $\mathrm{pH}$ obtenidos en la cámara del cátodo, así como en el segmento de suelo más próximo a este electrodo), se producirá la precipitación de los iones metálicos contaminantes, creándose una zona local de baja conductividad eléctrica. Estos resultados fueron confirmados analizando la conductividad residual de los distintos fragmentos de suelo.
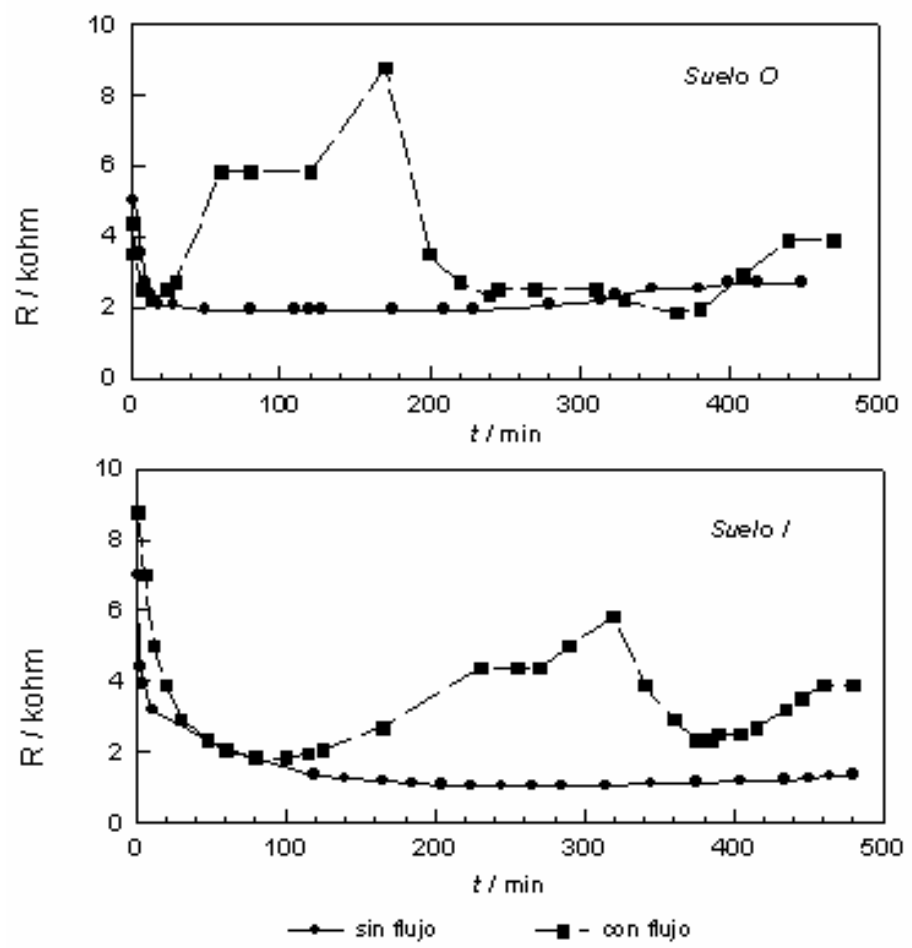

Figura 4. Influencia del flujo de lavado sobre la variación de la resistencia eléctrica con el tiempo de electrólisis.

Los valores de resistencia observados a tiempos mayores de tratamiento, podrían resultar de la mayor adsorción de iones que presenta el Suelo O, debido a su mayor contenido en materia orgánica.

Cuando se analiza el efecto de aplicar distintos valores de campo eléctrico puede interpretarse el comportamiento de la resistencia como una evidencia de que en los primeros instantes de la electrólisis, la corriente está siendo controlada por el transporte de iones hacia los electrodos. Cuando se aplica el mayor valor de potencial, se obtiene un valor alto de resistencia porque los iones deben movilizarse desde el segmento más próximo a las cámaras de los electrodos, 
atravesar el filtro que separa el suelo de las cámaras y dirigirse a la superficie del electrodo. De acuerdo a lo obtenido en este experimento, es conveniente emplear bajos potenciales en la celda de electro-remediación no solo porque se tiene una situación más homogénea con relación al valore de resistencia, sino que adicionalmente, se permite la participación del movimiento iónico en el interior de los poros del suelo.

La conveniencia o no de lavar continuamente las cámaras de los electrodos se refleja en los valores de resistencia obtenidos en cada caso. Nuevamente, esta variación debería ser analizada conjuntamente con los $\mathrm{pH}$ que presentan los distintos fragmentos de suelo al finalizar el tratamiento.

En términos generales puede afirmarse que no es conveniente el empleo de soluciones de lavado, ya que si bien se acentúa la influencia de los frentes ácido y alcalino generados sobre los electrodos, la capacidad reguladora que presentan los suelos resulta suficiente para evitar que estos frentes ingresen de manera significativa en los suelos, al menos para el tiempo analizado. Por otra parte, cuando no se emplea flujo de lavado, la resistencia eléctrica presenta una variación regular, pero por el contrario, al remover constantemente la solución que rodea al electrodo provoca que en determinados momentos se produzcan regiones de baja concentración iónica, con el consiguiente aumento abrupto de la resistencia.

A modo de comparación en la Fig. 5 se presentan unos resultados preliminares obtenidos en un experimento similar llevado a cabo en Tenerife con dos suelos de la isla. Nuevamente se observa que el empleo de flujo de lavado conduce a un aumento en la resistencia, incluso en los primeros instantes del tratamiento de remediación electroquímica.

Por lo anterior se puede concluir que las mejores condiciones experimentales para analizar el comportamiento de los suelos empleando tiempos cortos de tratamiento, están relacionadas con el empleo de bajos campos eléctricos sin remover la solución que rodea los electrodos. 

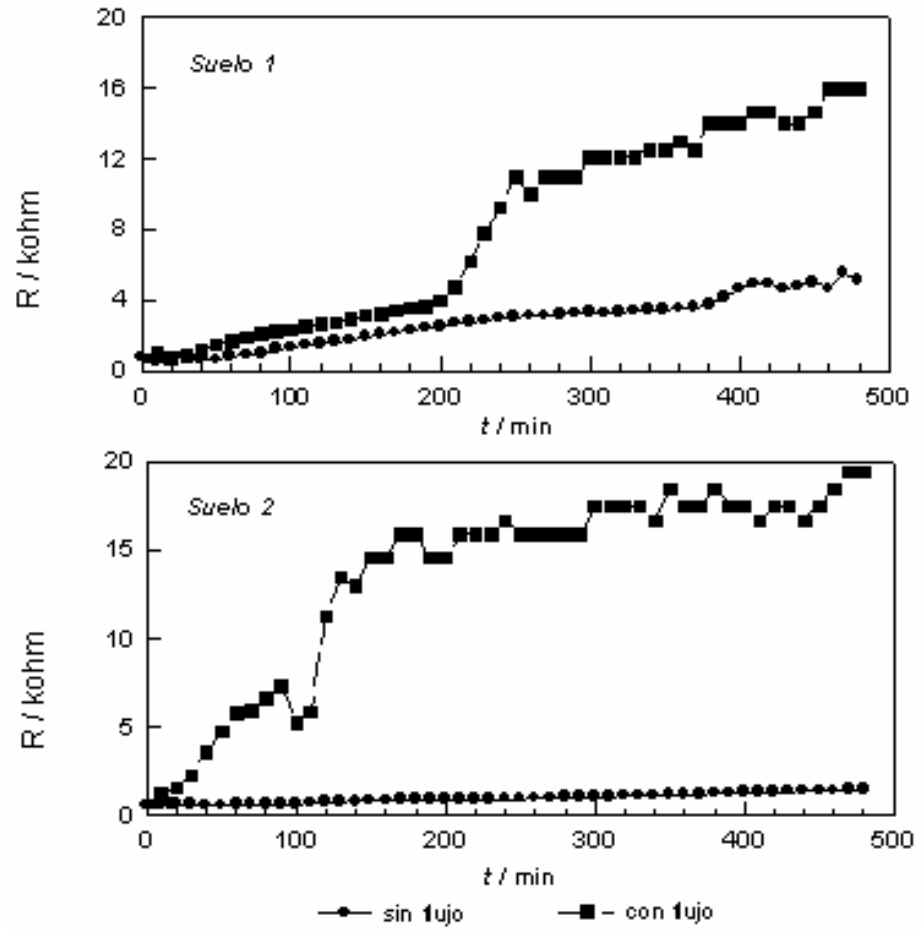

Figura 5. Influencia del flujo de lavado sobre la variación de la resistencia eléctrica con el tiempo de electrólisis. (Suelos 1 y 2, Tenerife, España).

\section{Agradecimientos}

Los autores agradecen a la Universidad de La Laguna (Proyecto Grupos Precompetitivo 1802440302) y a la Dirección General de Universidades del Gobierno de Canarias (Proyecto PI2003/138) por financiar parte de esta investigación. 


\section{Estudio de la Resistencia Electrica de Andisoles Sometidos a Tratamiento de Electro-Remediacion}

\section{Resumen}

Los estudios de la resistencia del suelo indican que este tipo de medidas, simultáneas al tratamiento de descontaminación, permiten de una manera rápida analizar el comportamiento de los distintos suelos bajo estudio, especialmente cuando no se aplica campo eléctrico.

La variación observada en la primera etapa de la electro-remediación, está relacionada con el contenido iónico en los segmentos adyacentes a los electrodos.

El empleo de un flujo de lavado facilita la formación de "baches iónicos" en las cercanías de los electrodos

El suelo con mayor contenido en materia orgánica, presenta una menor resistencia en la primera etapa del tratamiento.

El seguimiento de la corriente de electrólisis sin el empleo de flujo de lavado permitiría, conjuntamente con los demás parámetros experimentales, evaluar las características de los suelos en experimentos de relativamente corta duración

Palabras clave: andisoles, corriente de electrólisis, resistencia eléctrica, electroremediación, metales pesados.

\section{Referencias}

1. R.F. Probstein, R.E. Hicks, Science 260 (1993) 498.

2. A.B. Ribeiro, E.P. Mateus, L.M. Ottosen, G.B. Nielsen, Environm. Science Technology 34 (2000) 784.

3. L.M. Ottosen, H.K. Hansen, C.B. Hansen, J. App. Electrochem. 30 (2000) 1199.

4. M.M. Page, C.L. Page, J. Environmental Eng. (2002) 206.

5. $\quad$ M.V. Vázquez, D.A. Tobón Q., Afinidad 58 (2001) 437.

6. M.V. Vázquez, D.A. Tobón Q., F.L. Gutiérrez, Afinidad 59 (2002) 601.

7. J.A. Rodríguez, M.V. Vázquez, Universitas Scientiarum 8 (2003) 75.

8. M.V. Vázquez, F. Hernández-Luis, Innovación 15 (2003) 45.

9. K. Rajeshwar, J. Ibañez, "Environmental Electrochemistry, Fundamentals and Applications in Pollution Abatement”, Academic Press, California, (1997), pp 449-452.

10. F. Wenner, Bull. National Bureau of Standards 12 (4) 258, s 478-496; $(1915 / 16)$. 
11. M. de la Vega, A. Osella, E. Lascano, J. Appl. Geophysics 54 (2003) 97109.

12. A.J. Delaney, P.R. Peapples, S.A. Arcone, Cold Regions Science and Technology 32 (2001) 107-119.

13. M.V. Vázquez, F. Hernández-Luis, M. Lemus, C.D. Arbelo, Portug. Electroch. Acta 22 (2004) 387. 